\title{
Surgery for suspected neurogenic thoracic outlet syndromes: a follow up study
}

\author{
Michael Donaghy, Zelko Matkovic, Peter Morris
}

\begin{abstract}
Objectives-To assess the outcome of surgical treatment for thoracic outlet syndrome (TOS), and to compare the outcome in patients with and without an underlying cervical rib.

Methods-a heterogeneous group of 40 patients (33 women, seven men; aged 22-62 years) were evaluated 3 months to 20 years after surgery for suspected neurogenic TOS. Forty nine operations had been performed: cervical ribs were removed in 23 patients, together with fibrous band excision in nine. In the 17 without a cervical rib the thoracic outlet was decompressed by resection of the first thoracic rib in nine, and by other operations in eight.

Results-After surgery patients reported improved pain (33/36), sensory disturbance (30/35), hand muscle strength (14/27), and hand function (23/34). Postoperatively TOS recurred in two, and symptoms continued to progress in three patients in whom other diagnoses eventually emerged. Surgical complications were recorded in 10 patients, but were transient and did not result in permanent symptomatic sequelae.

Conclusions-Surgical treatment of suspected neurogenic TOS relieves pain and sensory disturbance $(90 \%)$, but is less effective for muscle weakness $(50 \%)$. Surprisingly, surgery relieved sensory and motor abnormalities to a similar degree in patients both with and without a cervical rib. Ideally, patients require early operation to forestall permanent hand muscle denervation, but, our retrospective analysis fails to identify any single preoperative diagnostic criterion for TOS, particularly in patients lacking a radiographic cervical rib.

(F Neurol Neurosurg Psychiatry 1999;67:602-606)
\end{abstract}

Keywords: cervical rib; thoracic outlet; surgery

of Surgery, University of Oxford, Radcliffe

Infirmary and John

Radcliffe Hospital, Oxford, UK

P Morris

Correspondence to: Dr Michael Donaghy. Department of Clinica Neurology, Radcliffe Infirmary, Oxford OX2 6HE UK. Telephone 00441865

224698; fax 00441865

790493

Received 29 June 1998 and in revised form

18 June 1999

Accepted 29 June 1999
The diagnosis of neurogenic TOS is relatively straightforward in the patient with pain and sensory disturbance predominantly in the ulnar forearm and hand, aggravated by use of the affected limb; associated with weakness and wasting of the small hand muscles particularly in the thenar eminence; a radiographic cervical rib; and neurophysiological studies confirming chronic postganglionic axonal loss, and excluding focal mononeuropathy. ${ }^{45}$ Many patients with suspected neurogenic TOS, however, fail to exhibit such a clear cut clinical, neurophysiological and radiological picture. Indeed it is self evident that the full blown syndrome must go through a long stage of evolution when such features are only partially present and insufficient for definite diagnosis of TOS. Further, although soft tissue anomalies, including various fibrous bands and less commonly scalenus anterior/medius anomalies may be responsible for TOS, ${ }^{6-8}$ only the bony abnormalities (cervical rib, malunited clavicular fracture, abnormal first thoracic rib) are clearly demonstrated by preoperative radiography. Unfortunately, there is no single criterion upon which to base a diagnosis. Currently this depends on collating the symptoms, examination findings, and results of electrophysiological and radiological studies. However, dynamic factors related to posture are of importance in TOS for even the findings at surgical exploration or brachial plexus imaging, when gravity no longer depresses the shoulder girdle, may not provide a diagnostic gold standard.

Despite its extensive history the surgical treatment of thoracic outlet syndrome remains controversial. Various operations have been advocated since Coote's pioneering removal of a cervical rib in 1861, with the transaxillary route, ${ }^{9}$ and the supraclavicular route ${ }^{10}$ being the commonest surgical approaches to the thoracic outlet in current practice. Although surgery is said to relieve pain, paraesthesia, and reduce muscle weakness in patients with full blown neurogenic TOS, the indications for, and the outcome of, surgery remain unclear for patients with a less clear cut clinical and radiological picture. ${ }^{43}$ None the less, the question of operative decompression needs to be considered in those patients in whom TOS can only be suspected, otherwise it would be impossible to forestall the ultimate development of irrecoverable axonal degeneration once full blown TOS has evolved. Some authors have reported a good or excellent postoperative outcome in up to $90 \%$ of these patients with suspected TOS, but often without providing a clear definition of the criteria for successful outcome..$^{9112}$ Others have highlighted disabling and permanent complications which may arise after surgery for TOS. ${ }^{3}{ }^{13}$ 
To enhance our understanding of how to manage suspected neurogenic TOS we have analysed the postoperative neurological outcome in a heterogeneous group of patients who underwent surgery in Oxford for suspected neurogenic thoracic outlet syndrome. In particular, we were interested to discover if the outcome differed significantly between those with and those without radiographic cervical ribs, thereby comparing a group likely to have definite TOS with a group in whom that diagnosis can only be inferred.

\section{Methods}

PATIENT POPULATION

From surgical department records we were able to contact 41 patients, only one of whom refused to take part, who had undergone surgery for suspected neurogenic thoracic outlet syndrome since 1973 .

\section{PREOPERATIVE FEATURES}

Medical records were reviewed for details of the patient's presentation and the findings on general, vascular, and neurological examination. Plain cervical $x$ rays had been performed in all, thoracic outlet and/or cervical spine MRI in 15, cervical myelography in four, and subclavian angiography in 11 . Routine diagnostic neurophysiological studies had been conducted preoperatively in 29 patients by various clinical neurophysiologists: concentric needle EMG of small hand muscles, median and ulnar motor conduction, and median and ulnar sensory nerve action potentials (SNAPs) had been carried out in all; $\mathrm{F}$ response and deep tendon reflex latencies had been determined in 25 .

SURGERY

Surgery had been performed by eight different consultant surgeons; 36 of the 49 thoracic outlet operations were carried out by one (PM). Surgery was performed by the supraclavicular approach in 34 patients. The scalenus anterior was divided to expose the subclavian artery and

Table 1 Preoperative features in suspected neurogenic thoracic outlet syndrome

\begin{tabular}{|c|c|c|}
\hline & $\begin{array}{l}\text { With cervical rib } \\
(n=23)\end{array}$ & $\begin{array}{l}\text { Without cervical rib } \\
(n=17)\end{array}$ \\
\hline \multicolumn{3}{|l|}{ Presenting symptoms: } \\
\hline Age at symptom onset (y (median)) & $10-57(35)$ & $21-58(31)$ \\
\hline Duration of symptoms (median) & Weeks-20 y (11 months) & 3 months -10 y $(2 \mathrm{y})$ \\
\hline Arm pain & 21 & 15 \\
\hline Arm sensory disturbance & 20 & 15 \\
\hline Hand weakness & 15 & 12 \\
\hline Impaired hand function & 20 & 14 \\
\hline Vascular symptoms & 14 & 5 \\
\hline \multicolumn{3}{|l|}{ Preoperative examination findings: } \\
\hline Decreased sensation & $14 / 23$ & $10 / 17$ \\
\hline Reduced muscle strength & $14 / 23$ & $11 / 17$ \\
\hline Muscle wasting & $10 / 23$ & $7 / 17$ \\
\hline Reproduction of sensory disturbance & & \\
\hline by rolling the brachial plexus & $7 / 7$ & $5 / 5$ \\
\hline Positional obliteration of radial pulse & $12 / 22$ & $12 / 15$ \\
\hline Subclavian bruit & 4 & 3 \\
\hline \multicolumn{3}{|l|}{ Neurophysiological studies: } \\
\hline Normal results & $1 / 15$ & $2 / 14$ \\
\hline Decreased F5 SNAP & $9 / 15$ & $10 / 14$ \\
\hline Denervation of APB or FD 10 & $14 / 15$ & $10 / 14$ \\
\hline Denervation of forearm flexor muscles & $2 / 6$ & $1 / 3$ \\
\hline Prolonged $\mathrm{F}$ waves & $6 / 13$ & $7 / 12$ \\
\hline Prolonged wrist flexor reflex latency & $2 / 4$ & $6 / 11$ \\
\hline
\end{tabular}

$\mathrm{SNAP}=$ Sensory nerve action potential $\mathrm{APB}=$ abductor pollicis brevis $; \mathrm{FD} 10=$ first dorsal interosseous muscle. lower trunks of the brachial plexus. Fibrous bands, if present, were divided, and cervical ribs or the first thoracic rib removed piecemeal with bone nibblers as far posteriorly as possible to leave the brachial plexus and subclavian artery "free" in the thoracic outlet.

The infraclavicular approach for first rib resection and the brachial plexus were retracted upwards in six patients exposing the first rib, which was excised with bone nibblers to a point well behind the brachial plexus.

POSTOPERATIVE NEUROLOGICAL ASSESSMENT The postoperative review at 3 months to 20 years (median 2 years) after surgery was by a neurologist who had not been involved in their preoperative management except in three cases. A full history was obtained including details of the presenting features: presence of arm pain, paraesthesia, numbness, weakness, wasting, vascular symptoms and loss of hand function; duration of symptoms; relieving and aggravating factors; and the effect on activities of daily living, employment, and leisure. The perioperative course, the nature and effect of complications, and the progress after discharge from hospital were ascertained. A full neurological examination of the limbs was undertaken. The vascular assessment involved palpation of upper limb pulses, checking for positional radial pulse obliteration, sphygmomanometer measurement of blood pressure in both arms, and auscultation for supraclavicular bruits. The outcome after surgery was judged according to the presence of postoperative complications, symptomatic relief, alteration in examination findings, and significant changes in disability.

\section{Results}

PREOPERATIVE FINDINGS

Table 1 shows preoperative findings. Presenting symptoms were similar in the two groups, exclusively or predominantly unilateral and left sided in 23 patients, right sided in 15, and bilateral in two; 32 were right handed. Each patient complained of arm pain, pain in the shoulder, forearm, or hand or sensory disturbance generally, paraesthesiae, in the ulnar aspect of the forearm or hand, or both. These sensory symptoms were typically intermittent and aggravated by use of the affected limb. A combination of these symptoms led to the frequent complaint (34) of impaired hand function interfering with manual tasks; in nine this restricted leisure activities or interfered with employment. Vascular symptoms such as changes in hand colour were never the dominant complaint.

The principle preoperative clinical features suggestive of TOS were pain or sensory disturbance in the ulnar forearm, with weakness of intrinsic hand muscles or flexor digitorum profundus.

Electrophysiology was abnormal in 26 out of 29 patients. Most commonly a reduced fifth digit sensory nerve action potential was associated with chronic partial denervation of small hand muscles. Plain cervical spine $x$ rays demonstrated bilateral cervical ribs in 16 patients, unilateral cervical ribs in five, elongated $\mathrm{C} 7$ 
Table 2 Complications of surgery

\begin{tabular}{lll}
\hline Patient age,sex & Operation & Complication \\
\hline $45, \mathrm{~F}$ & $\begin{array}{l}\text { Bilateral cervical rib resection and partial left } \\
\text { first rib resection }\end{array}$ & Bilateral shoulder girdle pains. Urinary tract infection \\
& $\begin{array}{l}\text { Bilateral cervical rib resection } \\
\text { Right cervical rib and fibrous band resection }\end{array}$ & $\begin{array}{l}\text { Shoulder girdle pains. Asymptomatic pneumothorax } \\
\text { Shoulder girdle pains, increased hand weakness } \\
\text { (traction injury), phrenic nerve palsy }\end{array}$ \\
$54, \mathrm{~F}$ & Left cervical rib resection & $\begin{array}{l}\text { Left basal atelectasis } \\
\text { Scar neuroma }\end{array}$ \\
$45, \mathrm{~F}$ & Left cervical rib resection & Increased hand weakness (traction injury) \\
$19, \mathrm{~F}$ & Left cervical rib resection & Shoulder girdle pains, phrenic nerve palsy \\
$47, \mathrm{~F}$ & Right first thoracic rib resection & Bronchitis \\
$39, \mathrm{~F}$ & Right first thoracic rib resection & Thoracic duct injury (lymph leakage settled in 48 hours) \\
$49, \mathrm{M}$ & Division of left fibrous band & Pleurisy \\
$24, \mathrm{~F}$ & Scalenotomy. & \\
$32, \mathrm{M}$ & & \\
\hline
\end{tabular}

transverse process in five, and a large anomalous first thoracic rib in one. Magnetic resonance imaging of the brachial plexus, carried out in 14 patients, showed distortion of the lower brachial plexus and cervical spine or C8-T1 nerve roots in 10 and, additionally, helped to exclude compressive cervical radiculopathy. Myelography and cervical spinal canal MRI in five patients were normal. Poststenotic subclavian artery dilatation or occlusion of the subclavian artery on abduction of the arm was detected in nine of the 11 patients who underwent angiography.

SURGICAL FINDINGS

In patients with a radiographic cervical rib, the thoracic outlet was explored by the supraclavicular route. Brachial plexus distortion or stretching was evident at operation in 17 out of 23 patients. In nine patients, a fibrous band extended from the tip of the cervical rib to the region of the scalene tubercle of the first thoracic rib; this was excised along with the cervical rib. To obtain adequate plexus decompression in two patients, a portion of the first thoracic rib as well as the cervical rib had to be removed. In two patients with bilateral symptoms both cervical ribs were excised during a single operation. In two patients the other cervical rib was removed 2 and 4 years later when symptoms had developed in the other arm.

In the 17 patients without a cervical rib a variable portion of the first thoracic rib was removed in nine, scalenotomy performed in five, elongated $\mathrm{C} 7$ transverse process excised in two, and a fibrous band extending from the scalenus medius to the first thoracic rib excised in one. In the nine patients who underwent first

Table 3 Outcome after surgery

\begin{tabular}{lll}
\hline & $\begin{array}{l}\text { With cervical } \\
\text { rib }(n=23)\end{array}$ & $\begin{array}{l}\text { Without cervical } \\
\text { rib }(n=17)\end{array}$ \\
\hline Change in symptoms: & $7 / 21$ & $5 / 15$ \\
$\quad$ Improved pain & $13 / 21$ & $8 / 15$ \\
$\quad$ Complete resolution of pain & $17 / 20$ & $13 / 15$ \\
Improved sensory disturbance & $8 / 15$ & $6 / 12$ \\
Improved muscle strength & $15 / 20$ & $8 / 14$ \\
Improved function & $8 / 14$ & $6 / 11$ \\
Improved power (on examination) & 11 & 7 \\
Neurological examination: & 7 & 5 \\
$\quad$ No motor or sensory abnormalities & 4 & 2 \\
Reduced sensation in the ulnar hand or forearm & & $6(5)$ \\
Other sensory abnormalities & $8(6)$ & $5(4)$ \\
Weakness or (wasting) of: & $6(5)$ & $7(3)$ \\
$\quad$ APB & $8(6)$ & 2 \\
$\quad$ FD10 or ADM & 5 & \\
Finger flexors & &
\end{tabular}

$\mathrm{APB}=\mathrm{Abductor}$ pollicis brevis; $\mathrm{ADM}=$ abductor digiti minimi; FD10=first dorsal interosseous muscle. thoracic rib resection anatomical anomalies responsible for neural distortion were noted in five: anomalous scalenus muscles in three, a very large anomalous first thoracic rib in one, and an abnormally long T1 nerve root angulated over the first thoracic rib in one. In one of the two patients in whom a prominent C7 transverse process was excised, a fibrous band running from the scalenus medius to Sibson's fascia was also divided. In five patients in whom no plexus distortion was evident at operation surgery was limited to scalenotomy (division of scalenus anterior).

SURGICAL COMPLICATIONS

Table 2 shows surgical complications. Thirty five patients underwent 43 operations by the supraclavicular approach. Asymptomatic right phrenic nerve injury occurred in two but none had damage to the long thoracic nerve. Transient increase in hand weakness, in two patients, was attributed to traction injury of the brachial plexus. Three patients experienced severe postoperative shoulder girdle pain which was controlled by oral analgesics and resolved after 1-3 months. All patients regarded the scar as cosmetically acceptable. Thoracic outlet decompression by first thoracic rib resection was performed through an infraclavicular approach in six patients with complications in two: severe but transient shoulder girdle pain in one and bronchitis in the other.

\section{POSTOPERATIVE SYMPTOMS}

Table 3 shows postoperative symptoms. At the neurological follow up assessment patients most often reported a favourable postoperative outcome. Appreciable symptom amelioration occurred a few days to 1 year (median, 1 month) after surgery. Arm pain had significantly diminished or disappeared in 33 of 36 patients. Thirty of 35 patients reported significant improvement of other sensory disturbance. Twenty three patients reported an improvement in hand function, including eight of the patients with muscle wasting. Reports of improved muscle strength or a clear cut improvement in power on examination occurred in about $50 \%$ of patients with preoperative muscle weakness. Established muscle wasting never improved.

The neurological examination was normal in 18 patients. Variable combinations of residual weakness or wasting of the small hand muscles or the forearm finger flexors were found in 17 . Notably, involvement of the flexor digitorum 
profundus was as common as abnormalities of the abductor pollicis brevis or the first dorsal interosseous. The abnormalities on sensory examination most often consisted of a blunting of pinprick sensation and light touch along the ulnar aspect of the hand and forearm in 12 . Other sensory changes were detected in six including a more extensive impairment of sensation in the arm.

NEUROLOGICAL PROGRESSION OR RECURRENCE POSTOPERATIVELY

Weakness and wasting of the small hand muscles continued to progress after surgery in three patients. In two without cervical ribs a diagnosis of chronic asymmetric spinal muscular atrophy emerged several years after first thoracic rib resection. In retrospect neither patient had experienced arm pain, and their modest sensory disturbance was overshadowed by prominent muscle weakness. In the third patient a foramen magnum decompression was carried out 15 months after cervical rib resection when the presence of syringomyelia and an Arnold-Chiari malformation was established.

Five patients developed recurrent neurological symptoms in the previously affected arm 3 months to 10 years after operation. Multiple recurrences of TOS occurred in a patient with Melkersson-Rosenthal-Miescher syndrome, which includes a tendency to fibrosis in the affected regions: a periosteal regrowth of the first thoracic rib was resected a year after the initial surgery and, on two further reoperations for recurrence of symptoms and signs, fibrous tissue which was distorting the brachial plexus was removed. Distortion of the brachial plexus by scar tissue was the cause of recurrence of TOS in another patient; symptoms recurred 10 years after cervical rib resection and resolved after excision of the fibrous tissue. In three other patients recurrence of symptoms occurred at 3 months, and 2 and 3 years after surgery: despite MRI studies in all three and neurophysiological testing in one a clear cause for the symptoms was not established; however, these "new" symptoms were less troublesome and produced less disability than the preoperative disturbance.

\section{Discussion}

The efficacy of surgical treatment for neurogenic TOS is debated. ${ }^{14}{ }^{15}$ In advanced forms of neurogenic TOS, resection of the underlying cervical rib and/or fibrous band relieves pain and paraesthesia in most patients, but usually does not alter the motor deficit significantly. ${ }^{4}$ Thus, surgery in such patients will not allow a useful recovery of muscle function and merely prevents further progression of the weakness and wasting. On the other hand, studies of patients without the "classic" full blown or advanced form of TOS have reported surgical success rates as high as $80 \%-90 \%$ without clear definition of the criteria on which a "good" or "excellent" outcome is based. ${ }^{9}{ }^{11} 12$ Moreover, some patients who had been described by their surgeon as having "improved" were subsequently judged not to have benefited from surgery when reviewed by a different observer. ${ }^{16}$ Thus, there was a clear need for a study of sur- gical outcome conducted by independent neurologists which would also evaluate the influence of preoperative severity and the presence of features such as a radiographic cervical rib on that outcome.

We found a generally satisfactory outcome after surgical treatment in this heterogeneous group of patients with suspected neurogenic TOS. In particular, we were surprised to find near identical surgical outcomes in the patients with and without cervical ribs, given that this anatomical variation might be regarded as considerably strengthening the likelihood of definite TOS. Pain and sensory disturbance improved in $90 \%$ of the patients, whereas improved muscle strength and hand function were reported by half. Muscle wasting never resolved, which suggests that early surgery may prevent irreversible denervation of hand muscles. Postoperative recurrence of TOS has been previously described ${ }^{17}$ and special circumstances prevailed in our two patients. The continued progression of upper limb symptoms in three patients was due to an alternative diagnosis from TOS. The postoperative outcome in our patients was not obviously influenced by the surgical approach (either supraclavicular or infraclavicular); the type of underlying anomaly (cervical rib, prominent C7 transverse process, anomalous first thoracic rib, fibrous bands, or abnormal insertion of scalenus muscles); or by the presence of visible brachial plexus distortion at operation.

We regard the anterior supraclavicular approach $^{10}$ as the operation of choice in patients with suspected neurogenic TOS who require surgical treatment. It provides the best exposure of the neurovascular bundle, cervical ribs, and fibrous bands and can be used for first rib resection. Its disadvantages include the risk of damage to the long thoracic or phrenic nerves and the presence of a cosmetically undesirable scar. ${ }^{1819}$ The transaxillary route ${ }^{920}$ is the most popular approach to the thoracic outlet in the United States: it leaves a small hidden scar, requires little dissection, but involves strenuous abduction of the arm, leaving the brachial plexus vulnerable to traction injury, and haemostasis may prove difficult if there is intraoperative damage to the subclavian vessels. Although the infraclavicular approach $^{21}$ provides excellent access to the anterior two thirds of the first rib it does not, in general, allow visualisation of the anatomical abnormality and is not commonly practised. We recorded surgical complications in 10 of 40 patients but these did not give rise to long term or permanent symptoms or disability.

No single diagnostic criterion for TOS can be used in making the decision to offer a patient surgical exploration with a view to decompression of the thoracic outlet, and even the intraoperative findings may not be diagnostic. Helpful diagnostic symptoms include sensory disturbance in the C8 and T1 dermatomes, aggravation of pain, and paraesthesia by use of the affected arm, absence of nocturnal symptoms, and the presence of vascular symptoms when the subclavian artery is also involved. We regard the typical motor features of TOS to consist of 
weakness of the small hand muscles, particularly of the thenar eminence, and of the long finger flexors. Unfortunately these muscles are usually not tested in a suitably sensitive manner to detect mild weakness; we recommend that the examiner use his own flexor digitorum profundus or dorsal interosseous muscles to test the patient's terminal interphalangeal joint flexion, and finger abduction respectively. Although muscle weakness had not been recorded in all of our patients, we do not think that the examination had always been performed in the rigorous manner we advocate. Radial pulse obliteration by Wright's manoeuvre (hyperabduction and elevation of the arms) or Adson's test (lateral rotation of the neck and hyperinflation of the chest) is not very reliable as a diagnostic test because of "positive" results in many normal asymptomatic people. ${ }^{19}$ We attach considerable diagnostic importance to reproduction or precipitation of the sensory symptoms in the ulnar forearm by rolling the brachial plexus in the supraclavicular fossa from behind when the patient is standing with the arms hanging.

It is generally agreed that neurophysiological studies are important in diagnosing neurogenic TOS. ${ }^{5}$ They can exclude alternative diagnoses of common focal neuropathies such as carpal tunnel syndrome or ulnar nerve palsy. Electromyography shows denervation of forearm flexors and small hand muscles, and sensory nerve action potentials can confirm that the underlying lesion is postganglionic, and the rarely performed tendon reflex latencies may point to a proximal lesion. ${ }^{22}$ Unfortunately, preoperative neurophysiological studies had not been conducted in all patients, as our study group had been referred to the surgical services over many years, by a disparate group of physicians, and were sometimes only requested in the presence of a doubtful diagnosis. All three of our patients with normal electrophysiological results showed benefit from surgical treatment: resection of a large cervical rib in one; division of fibrous band from scalenus medius to the first thoracic rib in one; removal of the first thoracic rib in one. It should be noted that an ulnar nerve SAP which lies within the normal range could have decreased from a higher premorbid level, and should be compared with the SAP in the unaffected arm. There is little experience of measuring SAPs from the first thoracic dermatome on the upper forearm, which should be the earliest neurophysiological abnormality in brachial plexus compression from below; study of the medial cutaneous nerve of the forearm could prove useful in this regard. In one of our patients with TOS due to a cervical rib and band, sensory potentials from the digits were normal and symmetric but a response could not be obtained from the medial cutaneous nerve of forearm on the side of symptoms, whereas a normal response was easily obtained on the other side. Thus patients with normal conventional upper limb neurophysiological studies who otherwise meet the criteria for the diagnosis of TOS should not be excluded from being considered for surgical treatment if other indications are convincing.
The results of a retrospective study must by definition be regarded with caution. Unfortunately, the outcome remains unknown in a similar group of patients with TOS who have not undergone surgical treatment. Nevertheless, the results of our study do suggest that surgical decompression is highly likely to alleviate what can be disabling upper limb symptoms in patients with a wide range of abnormalities at the thoracic outlet. Although in patients with suspected neurogenic TOS who lack objective neurological findings, progression to muscle weakness and wasting has not been definitely established, ${ }^{3}$ it does seem likely that such progression will eventually occur in many as part of the natural evolution of TOS. Further, given that established motor abnormalities respond poorly to surgery for TOS, exploration should be considered in such patients before the irreversible denervation occurs, providing that conservative methods have failed, and the symptoms are disabling. The patient must recognise the possibility of complications and, particularly if there is no cervical rib, the exploratory nature of such an operation.

We thank Mrs Anne Richardson and Mrs Joanna Wilkinson for their patient and expert secretarial assistance, and Mrs Jackie Walton for help in identifying patients.

1 Wilbourn AJ. Brachial plexus disorders. In: Dyck PJ, Thomas PK, Lambert EH, et al, eds. Peripheral neuropathy. Philadelphia: WB Saunders, 1993:911-50.

2 Peet RM, Henriksen JD, Anderson TP, et al. Thoracic outlet syndrome. Mayo Clinical Proceedings Staff Meeting 1956;31: 281-7.

3 Wilbourn AJ. Thoracic outlet syndrome surgery causing severe brachial plexopathy. Muscle Nerve 1988;11:66-74.

4 Gilliatt RW, Le Quesne PM, Logue V, et al. Wasting of the hand associated with a cervical rib or band. 7 Neurol Neurosurg Psychiatry 1970;33:615-26.

5 Smith T, Trojaborg W. Diagnosis of thoracic outlet syndrome: value of sensory and motor conduction studies and quantitative electromyography. Arch Neurol 1987;44: and quanti-3.

6 Bonney G. The scalenus medius band. F Bone foint Surg Br 1965;47:268-72.

7 Berquist E, Hugosson R, Westerberg CE. A wasted hand: case with uncommon neurological and radiological features caused by a cervical band. F Neurol Neurosurg Psychiatry 1975;38:100-2.

8 Roos DB. Congenital anomalies associated with thoracic syndrome; anatomy, symptoms, diagnosis and treatment. Am $\mathcal{F}$ Surg 1976;132:771-8.

9 Roos DB. Transaxillary approach for the first rib resection to relieve thoracic outlet syndrome. Ann Surg 1966;16: 354-8.

10 Falconer MA, Li FWP. Resection of the first rib in costo-clavicular compression of the brachial plexus. Lancet 1962;i:59-63.

11 Kelly TR. Thoracic outlet syndrome. Ann Surg 1979;190: 657-62.

12 Wood VE, Twito R, Verksa JM. Thoracic outlet syndrome. Orthop Clin North Am 1988;19:131-46.

13 Cherington M. Surgery for the thoracic outlet syndrome? $N$ Engl f Med 1986;314:322.

14 Roos DB. The thoracic outlet syndrome is underrated. Arch Neurol 1990;47:327-8.

15 Wilbourn AJ. The thoracic outlet syndrome is overdiagnosed. Arch Neurol 1990;47:328-30.

16 Carroll RE, Hurst LC. The relationship of thoracic outlet syndrome and carpal tunnel syndrome. Clin Orthop 1982;164:149-53.

17 Roos DB. Recurrent thoracic outlet and carpal tunnel syndromes. In: Rutherford RB, ed. Vascular surgery. Philadelphia: WB Saunders, 1984:708-24.

18 Urschel HC, Razzuk MA. Management of the thoracic outlet syndrome. $N$ Engl F Med 1972;286:1140-3.

19 Lascelles RG, Schady W. The thoracic outlet syndrome. In: Matthews WB, coed. Neuropathies. Vol 51. In: Vinken PJ, Matthews WB, coed. Neuropathies. Vol 51 . In: Vinken PJ,
Bruyn GW, eds. Handbook of clinical neurology. Amsterdam: Bruyn GW, eds. Handbo

20 Roos DB. Experience with first rib resection for thoracic outlet syndrome. Ann Surg 1971;173:429-33.

21 Gol A, Patrick DW, McNeel DP. Relief of costoclavicular syndrome by infraclavicular removal of first rib: Technical note. F Neurosurg 1968;28:81-4

22 Portner MR. Electrophysiological measurements of the reflex activity in the diagnosis and localisation of organic nervous disease [MSc thesis]. Oxford: University of Oxford, 1980. 